\title{
BMJ Open FullFix: a randomised controlled trial of a telephone delivered transdiagnostic intervention for comorbid substance and mental health problems in young people
}

Zoe Walter (D) , ${ }^{1,2}$ Catherine A Quinn, ${ }^{1,2}$ Genevieve Dingle, ${ }^{1}$ Nina Pocuca (D) , Amanda L Baker, ${ }^{3}$ Alison Beck, ${ }^{3}$ Dominique De Andrade, ${ }^{1}$ Maree Toombs, ${ }^{4}$ Leanne Hides ${ }^{1,2}$

To cite: Walter Z, Quinn CA, Dingle G, et al. FullFix: a randomised controlled trial of a telephone delivered transdiagnostic intervention for comorbid substance and mental health problems in young people. BMJ Open 2021;11:e045607. doi:10.1136/ bmjopen-2020-045607

- Prepublication history and additional supplemental material for this paper are available online. To view these files, please visit the journal online (http://dx.doi.org/10.1136/ bmjopen-2020-045607).

Received 06 0ctober 2020 Accepted 15 September 2021

Check for updates

(c) Author(s) (or their employer(s)) 2021. Re-use permitted under CC BY-NC. No commercial re-use. See rights and permissions. Published by BMJ.

For numbered affiliations see end of article.

Correspondence to

Dr Zoe Walter;

z.walter@uq.edu.au

\section{ABSTRACT}

Introduction Transdiagnostic cognitive-behavioural therapy (CBT) targets common psychological factors that underlie multiple disorders. While transdiagnostic interventions are a promising new approach, limited research has evaluated these treatments within the alcohol and other drug (AOD) sector for young people with comorbid mental health symptoms. This project will examine the feasibility and preliminary efficacy of FullFix - a new risk-targeted transdiagnostic CBT telehealth programme for comorbid AOD and depression/anxiety disorders in young people. Secondary aims are to identify moderators and mediators of treatment outcomes, to determine how and why treatment is effective and who is most likely to benefit.

Methods/design Participants will be 130 young people (aged 16-35) accessing AOD services in Queensland, Australia, with comorbid mental health symptoms. They will be randomised to receive either the FullFix intervention plus standard AOD care or standard AOD care alone. Primary outcomes on AOD use and mental health symptoms will be reassessed at 6 weeks, 3 months, 6 months and 12 months, along with secondary outcomes of emotion regulation, social connectedness, perceived self-efficacy, coping skills and quality of life. The trial commenced on October 2018 and expected completion date is September 2021.

Ethics and dissemination Ethical approval for this trial was provided by the University of Queensland (\#2018001185). The results of the trial will be disseminated through publication in a peer-reviewed scientific journal, scientific presentations at conferences and distributed via a report and presentations to the partner organisation.

Trial registration number ACTRN12618001563257.

\section{INTRODUCTION}

Epidemiological and clinical studies consistently show that alcohol and other drug use
Strengths and limitations of this study

- This is a novel project using a transdiagnostic telephone-delivered intervention to target both risk and protective factors for alcohol and other drug (AOD) and anxiety/depression problems for young people.

- Telephone-delivered transdiagnostic cognitive-behavioural therapy could provide an accessible, efficacious and cost-effective treatment for addressing the significant issue of comorbidity in young people.

- The delivery of the intervention as an adjunct treatment to standard AOD care minimises disruption to routine practice.

- A diverse range of $\mathrm{AOD}$ treatments are likely to be delivered as 'standard care'.

Self-selection may result in people opting in or out depending on their preference for telehealth or traditional face-to-face counselling, potentially biasing results.

(AOD) and mental disorders frequently co-occur. $^{1-6}$ For instance, in a nationally representative survey of 8841 Australians (aged 16-85 years), one in three respondents with a past year substance use disorder also met criteria for an anxiety disorder, and one in five met criteria for an affective disorder. ${ }^{7}$ The high rate of co-occurring substance use and mental health disorders incur significant costs to society, and are a key challenge to policy and practice. Comorbidity is related to a more severe and chronic illness course, increased relapse risk, worse treatment outcomes, higher risk of suicide and greater social and functional impairment. ${ }^{8-11}$ This challenge is magnified in people accessing AOD services, who frequently present with additional psychosocial complexities, such 
as history of trauma, neglect, homelessness, criminal justice involvement and unemployment. ${ }^{12} 13$

High rates of comorbidity and associated treatment challenges are of particular concern in young adults. Mental health and substance use disorders are at their peak and the leading cause of disability and death worldwide in adolescence and young adulthood. ${ }^{14}{ }^{15}$ Further, although there is strong epidemiological evidence that $75 \%$ of AOD and mental health problems emerge before the age of 25 , treatment typically does not occur till many years later, ${ }^{16}{ }^{17}$ with one study finding an 18-year gap between problem identification and receipt of AOD treatment. $^{18}$

Current practice approaches often fail to address the complex patterns of comorbid mental health and AOD problems with which young people present. Although an integrated approach to the management of comorbidity is recommended, ${ }^{19-21}$ treatments that target comorbidity remain underutilised within AOD treatment services. ${ }^{22}$ FullFix, an integrated telehealth intervention for young people with comorbid AOD and mental health problems, was developed to address these treatment concerns.

\section{Telehealth interventions}

Few young people experiencing mental health problems, particularly AOD problems, access treatment. For instance, one study found fewer than one in four young people with substance use disorders had accessed health services in the past year. ${ }^{23}$ Young people face many barriers to accessing treatment, with stigma and shame being a key deterrent to help-seeking. ${ }^{24}$ Telephonedelivered (telehealth) interventions provide an innovative, youth friendly way of rapidly increasing treatment access to the $96 \%$ of young people who own a mobile phone. ${ }^{25}$ Previous studies in adolescents reported young people found telephone-delivered interventions more acceptable, convenient, flexible and less stressful than attending a clinic. ${ }^{26}{ }^{27}$ Further, meta-analyses have found that telephone-delivered psychological therapies, particularly cognitive-behavioural therapy (CBT), have promise in improving mental health outcomes. ${ }^{26}{ }^{28}{ }^{29}$ Within AOD treatment, telephone-delivered interventions have been shown to be effective for reducing risk of future AOD use and related harm in young people. ${ }^{30} 31$

\section{Transdiagnostic approaches}

Transdiagnostic interventions, which cut across diagnostic boundaries to target risk factors underlying multiple problems, are a promising new approach to the treatment of comorbid disorders. ${ }^{32} 33$ Theoretically, transdiagnostic approaches suggest that diverse mental health disorders have common latent factors, which develop from interactions between common genetic, biological, psychological and environmental vulnerabilities. ${ }^{34-39}$ Common identified transdiagnostic risk factors and protective factors for both AOD include (1) impulsive, sensation seeking and neurotic personality traits, ${ }^{40-44}$ (2) coping, selfcontrol and emotion regulation skills ${ }^{34} 364345-48$ and (3) social factors, such as social support or the social normative environment. ${ }^{3949}{ }^{50}$ Developing an intervention that targets these three key areas could provide more efficient and effective treatments for AOD and comorbid mental disorders.

There is a growing evidence-base demonstrating transdiagnostic approaches, including transdiagnostic $\mathrm{CBT}^{22}{ }^{51-53}$ mindfulness-based interventions ${ }^{54}$ and emotion-regulation focused interventions ${ }^{45}$ have positive outcomes for depression and anxiety disorders. However, a recent narrative review of transdiagnostic approaches for comorbid depression and AOD disorders concluded that while transdiagnostic treatment approaches have been developed for emotional disorders and anxiety disorders, there are limited programmes targeting AOD comorbidities. ${ }^{22}$ The authors conclude that despite the lack of trials examining transdiagnostic interventions for AOD, such process-based approaches hold promise, as treatments that target transdiagnotic factors-specifically, negative affect, anhedonia, rumination, experiential avoidance, emotion regulation and distress tolerancelead to improvements in depression and AOD use.

\section{Objectives of the study}

Overall, the evidence suggests that a transdiagnostic approach to addressing comorbid AOD and mental health problems has a strong theoretical basis with empirically derived treatment targets, and has the potential to improve clinical outcomes in clinically complex populations. Yet, there is a dearth of studies examining transdiagnostic interventions for young people with AOD use and comorbid problems. The current project aims to examine the feasibility and efficacy of a new risk and protective factor-targeted transdiagnostic CBT telehealth programme (FullFix) for comorbid AOD and mental health problems in young people delivered as an adjunct to standard AOD care, compared with standard AOD care alone. Feasibility and outcomes are assessed at 6 weeks (approximately mid-treatment), 3 months (posttreatment), 6 months and 12 months postbaseline.

Secondary aims are to identify moderators and mediators of treatment outcomes, to determine how and why treatment is effective and who is most likely to benefit. This information will be used to further refine FullFix to make it more targeted and effective.

We hypothesise that individuals receiving FullFix plus standard AOD treatment will achieve significantly greater improvements on the primary outcomes of AOD use and mental health as well as the secondary outcomes of mental well-being and functioning outcomes, compared with individuals receiving standard AOD treatment alone at 3-month, 6-month and 12-month postbaseline.

\section{METHODS AND ANALYSIS Study design}

This multicentre randomised controlled trial (RCT) will compare the efficacy of a transdiagnostic CBT 


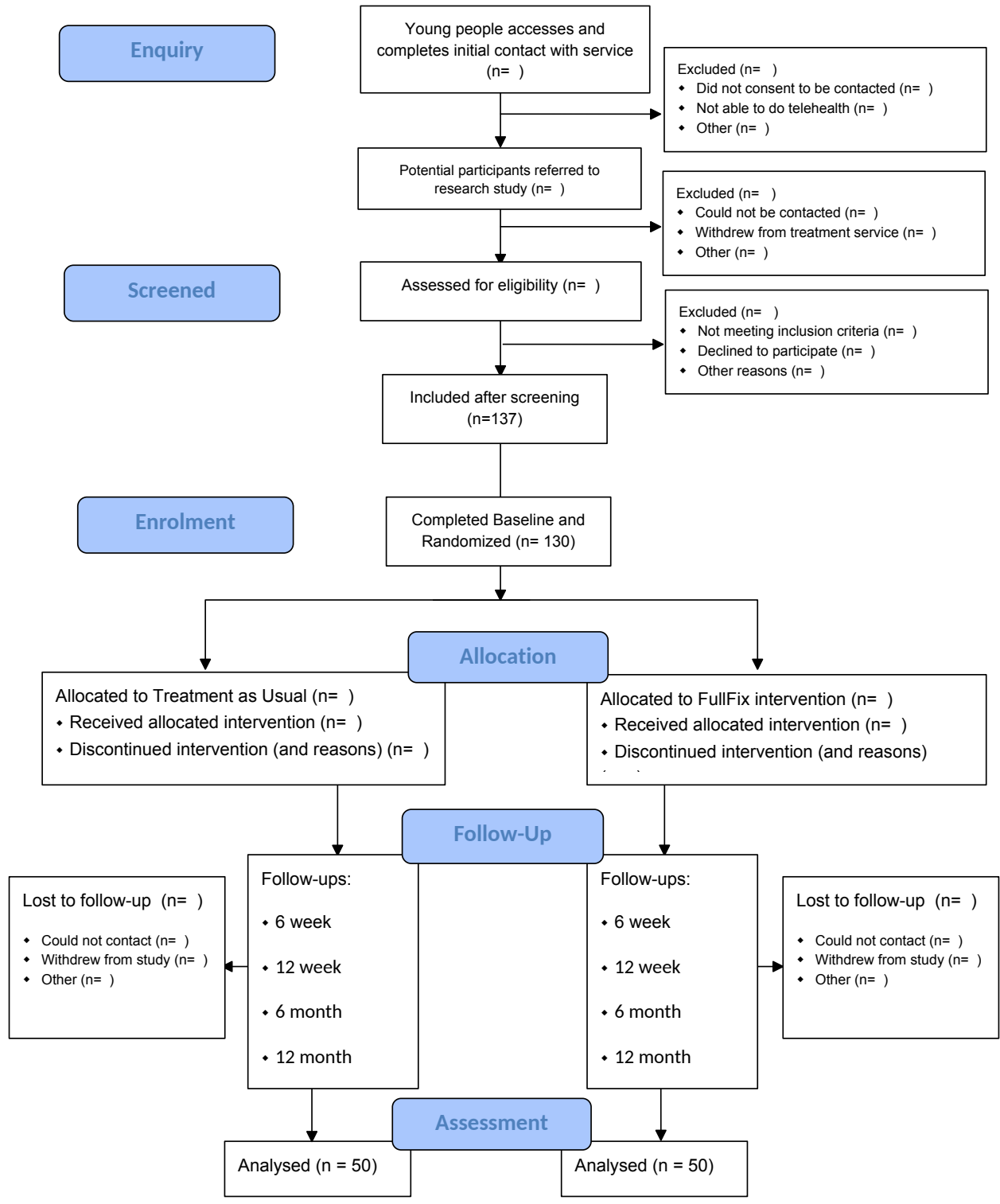

Citation: Eldridge SM, Chan CL, Campbell MJ, Bond CM, Hopewell S, Thabane L, et al. CONSORT 2010 statement: extension to randomised pilot and feasibility trials. BMJ. 2016;355.

Figure 1 Projected CONSORT diagram, showing expected retention and loss. CONSORT, Consolidated Standards of Reporting Trials.

intervention (FullFix) delivered as an adjunct to standard care, compared with standard care alone for young people accessing inpatient and outreach AOD services. The trial will also assess the feasibility to deliver the FullFix intervention in these settings. The trial is registered (ACTRN12618001563257) and follows Standard Protocol Items: Recommendations for Interventional Trials research protocol guidelines (see online supplemental materials) as well as Consolidated Standards of Reporting Trials guidelines (see figure 1).

\section{Study settings}

Young people (16-35 years) will be recruited from Lives Lived Well (LLW) AOD treatment services. This includes seven services providing outreach and on-site services and a residential inpatient treatment service in Queensland, Australia. Approximately $50 \%$ of clients referred to these services are presenting for treatment for the first time.

\section{Eligibility criteria}

Inclusion criteria are: (1) 16-35 years of age; (2) seeking help for current AOD use from participating LLW services; (3) Alcohol, Smoking and Substance Involvement Screening Test (ASSIST ${ }^{55}$;) score of either $\geq 10$ for alcohol use, or $\geq 4$ for illicit drug use; (4) a score $\geq 10$ for either the Patient Health Questionnaire-9 (PHQ$9^{56}$;) or the Generalised Anxiety disorder $\left(\mathrm{GAD}-7^{57} ;\right.$ ), or a score of $\geq 3$ on the Primary-Care Post-traumatic Stress Disorder Screen ${ }^{58}$; and (5) access to mobile phone technology. Exclusion criteria are: (1) current serious medical 
problem or traumatic injury; (2) not fluent in spoken or written English; (3) unmodified hearing impairment; (4) current acute high suicide risk (intent and plan) as assessed by the treating clinician; (5) current psychosis or diagnosed schizophrenia that is currently being treated with antipsychotics; (6) history of traumatic brain injury or organic brain disease that interferes with treatment delivery and (7) currently in acute alcohol or drug withdrawal. There are no restrictions on concomitant care or other interventions that participants can access or take part, in addition to the study trial.

\section{Sample size calculations}

Medium effects ( $\mathrm{f}=0.25)$ are anticipated based on our previous CBT trial. ${ }^{59}$ With power set at 0.90 , and alpha of $0.05,52$ participants in each group, or a total sample of 104 , is required. We predict a $20 \%-30 \%$ attrition rate based on our previous work and will therefore aim to recruit a minimum of 132 (20\% attrition) and up to 149 (30\% attrition) participants. All participants who are randomised to a treatment condition will be included in the intent-to-treat analysis.

\section{Recruitment and follow-up procedures}

Participants will be recruited from new clients entering the service or beginning a new programme at a participating site. After enrolling in a treatment programme, LLW staff will provide potential participants (clients aged 16-35 seeking assistance for AOD use) with brief information about the research, and then obtain their verbal or written consent to be contacted by a researcher, as part of a standard consent process. Programme enrolments will be monitored weekly by a trained research assistant and the project manager to ensure the protocol is being followed. A research assistant will directly contact LLW staff to follow-up on referrals that do not have consent status (yes/no) recorded. Trained research assistants with a minimum honours degree in psychology will then contact the consenting client within 3 days to complete the screening measures and obtain full informed consent. Participants meeting inclusion criteria will be sent a survey link to complete the baseline assessment online. Those that complete the baseline survey will be randomised to receive either FullFix+standard AOD care or standard AOD care only.

Participants will be asked to complete follow-up surveys online at 6 weeks, 3 months, 6 months and 12 months post-baseline. All participants will be followed up for intent-to-treat purposes, regardless of treatment completion. Reminders to complete the survey will be sent to participants by research assistants blind to treatment allocation, and will be followed up over the phone if the survey is not completed within 1 week. A follow-up protocol has been developed, which includes a schedule for when to send reminders and via what modality (email, text messages to mobile devices, social media, phone-calls and locator phone-calls and text messages). Participants will be reimbursed AUD $\$ 20$ for completing the baseline and each follow-up survey.

Recruitment commenced September 2018, with enrolment of the first participant occurring in October 2018. Recruitment was concluded in July 2020, with the last participant enrolment occurring in August 2020. Data collection is due to be finished in September 2021.

\section{Randomisation}

A randomisation sequence has been created using statistical software $R$ with the package 'blockrand' ${ }^{60}$ The randomisation sequence was stratified by service area (six sites) and age (16-20, 21-25, 26-30, 31-35), with a 1:1 allocation using random block sizes of 4,6 and 8 to randomise participants into (1) FullFix +standard AOD treatment or (2) standard AOD treatment alone. The randomised sequence is contained in a password protected spreadsheet. Randomisation sequence and allocation will be conducted by independent research assistants not involved in recruitment or data collection. Allocation will be communicated to the chief investigator and LLW staff to assist with treatment planning.

\section{FullFix intervention}

FullFix is an eight-session telehealth transdiagnostic CBT intervention which targets the risk and protective factors common for comorbid AOD and depression/ anxiety problems. It incorporates the evidence based QuikFix personality-specific coping skills brief intervention. ${ }^{26}$ QuikFix is a two session intervention comprising assessment feedback/information, motivational interviewing $(\mathrm{MI})$, psychoeducation and coping skills training targeting the participant's predominant maladaptive personality traits related to problematic AOD use. It has been found to result in larger reductions in the number of standard alcohol drinks consumed at both 1 and 12 months follow-up among young people with alcohol related injuries and illnesses, compared with assessment feedback/information or MI alone. ${ }^{30} 316162$ In the pilot RCT for QuikFix, although people who received QuikFix reported significantly reduced distress (on the Kessler Psychological Distress Scale (K10)), 88\% of participants nevertheless continued to report at least moderate distress at 1-month follow-up (K10 score $\geq 17$ ) ${ }^{61}$ suggesting that people with mental health comorbidity may benefit from a more intensive intervention. This is addressed in FullFix which is designed to be delivered using a stepped care framework. Specifically, all participants are first provided with the QuikFix brief intervention (sessions 1 and 2 of FullFix), followed by sessions focused on building protective factors and strengths (session 3 ) and teaching participants a self-regulation framework for implementing coping skills in different situations (session 4).

After these four core modules, young people are offered up to four additional, optional, sessions. The order of the four additional sessions is flexible and are determined in collaboration with the client. These modules target (1) positive and negative emotional 
awareness and regulation, (2) healthy behaviours and behavioural regulation, (3) cognitive regulation and (4) social connectedness and support. These sessions are tailored to the individual's specific risk and protective factor profile by a trained psychologist using a caseformulation approach. All young people are offered the opportunity to receive these additional four sessions, regardless of their initial response to FullFix. Modules are delivered individually in $30-50 \mathrm{~min}$ telephone therapy calls for up to 8 weekly or fortnightly sessions, which may be broken into two shorter calls per week if the participant prefers. Table 1 provides a summary of all intervention modules.

An MI style (eg, empathically amplifying the person's own concerns) ${ }^{63}$ is used throughout FullFix to: (1) build readiness and commitment to make a change (including pros and cons of current use and change); (2) negotiate change goals; and (3) develop a plan for change each session, with a focus on building self-efficacy to achieve change. Development of the plan includes developing specific, measurable, and achievable goals within a timeframe, discussing support and role-models for achieving goals, recalling past successes of behaviour change, personal strengths or strategies for achieving goals, potential barriers or challenges to implementing the plan (and ways to overcome barriers), and the reasons underlying the goals.

\section{Standard AOD care}

The control group receives standard AOD care in accordance with standard practice at the participating site. Details of episodes of care and session case notes will be recorded by LLW clinicians. Frequency of sessions, duration, modality (phone or face-to-face) and type of session (eg, counselling, case management) will be measured for each participant. An independent researcher will conduct a file audit to check the reliability of the episodes of care data for a random sample of $20 \%$ of participants to determine inter-rater reliability. Personalised assessment feedback on the participant's AOD use and related physical, psychological and social consequences will also be sent to the usual care clinician via email.

\section{Community services}

Community standard AOD care typically consists of case management and AOD counselling, delivered via phone or face to face by an LLW AOD clinician (typically an allied health professional with at least a bachelor's degree). A typical episode of care consists of 2-10 sessions. Usual care is not based on specific guidelines, nor does it have a prescribed number of sessions, frequency or duration of sessions; as this is determined on a case by case basis by the AOD worker. Typical treatment approaches used include CBT for substance use, MI, psychoeducation and counselling.

\section{Residential rehabilitation services}

Residential Rehabilitation standard AOD care typically consists of a 6-week treatment programme, with a maximum length of stay of 12 weeks, and telephone support by pretreatment/post-treatment AOD workers to assist with admission into the residential facility and the transition back to the community. The programme includes regular group sessions covering substance use (eg, triggers, cravings, building motivation to change, drug refusal skills), emotion regulation and management, grief and loss, developing a relapse prevention plan; one on one counselling/case management; as well as creativity, exercise, nutrition and relaxation activities.

\section{Patient and public involvement}

Consultations were conducted with clinicians and staff of the participating organisations to inform the study protocols and design. Patients were not involved in the design of the study or intervention. The project manager and relevant members of the research team meet regularly with team leaders and area managers of the services involved in the project, as well as monthly meetings with organisation management. At these meetings, feedback is invited on the conduct of the research. Adaptations to the protocol will be made, if necessary, based on this ongoing feedback. The measures assessing client feedback will be used and incorporated into future studies involving service users.

\section{Feasibility}

A number of outcomes will be used to assess feasibility. We will assess recruitment methods using the number of potential participants per week who are: eligible, approached, consented and who meet inclusion criteria. We will also assess the number of consenting participants who are willing to be randomised, the number who complete all baseline assessments within 14 days, and the number who complete follow-up assessments within 30 days of target date. Additionally, we will measure reasons for refusal or non-completion at all stages of research (screening, enrolment, randomisation and all follow-up time-points). To specifically assess feasibility of the intervention, we will record number of consenting participants who commence their randomly allocated treatment within 14 days, and sessions completed by participants.

\section{Measures}

The assessment schedule is presented in table 2.

\section{Demographics}

Participants will be asked to report their age, gender, occupation, years of education, relationship status, living arrangements (including postcode), ethnicity, family and personal history of mental and substance use disorders, history of traumatic brain injury or organic brain disease. 


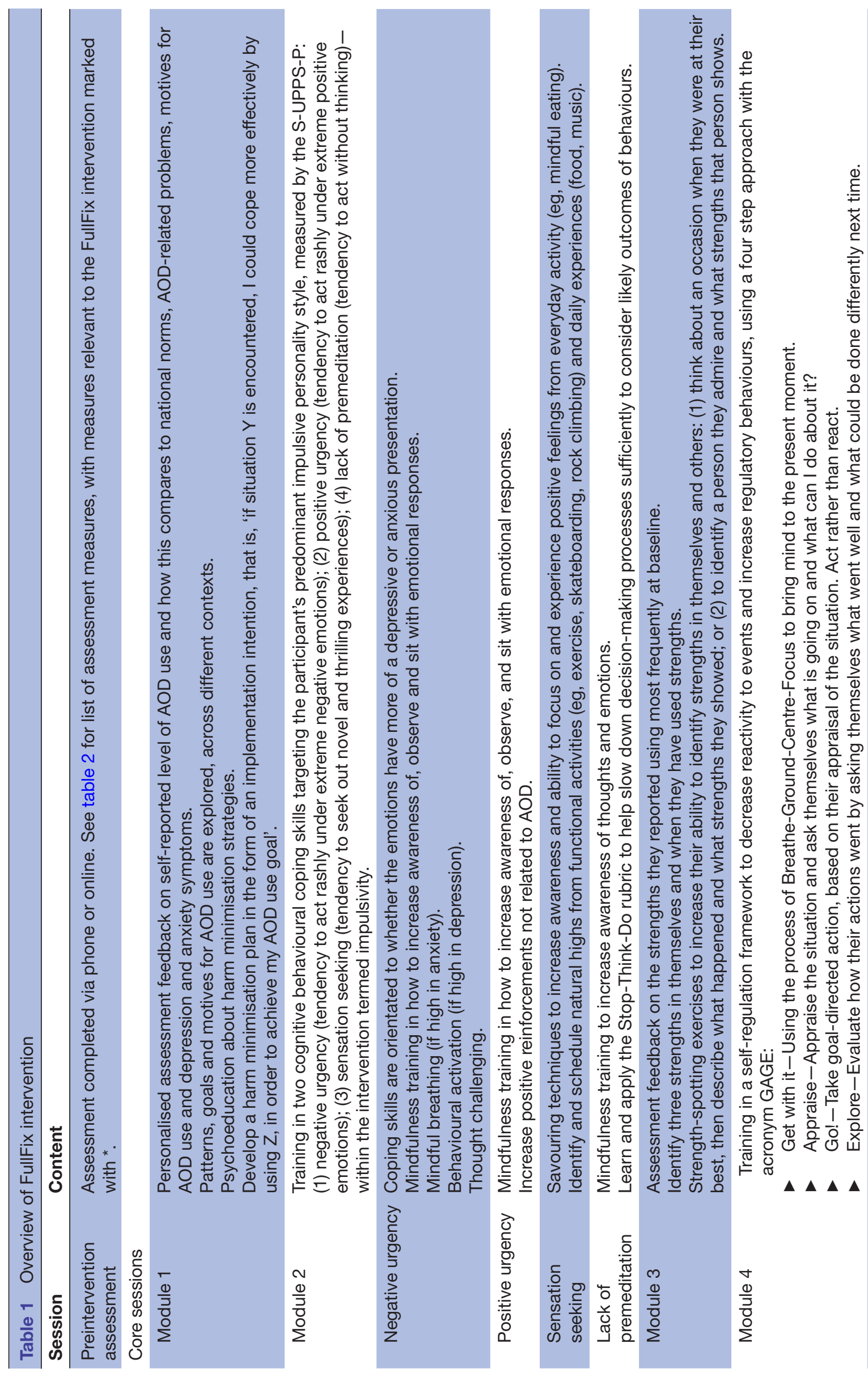




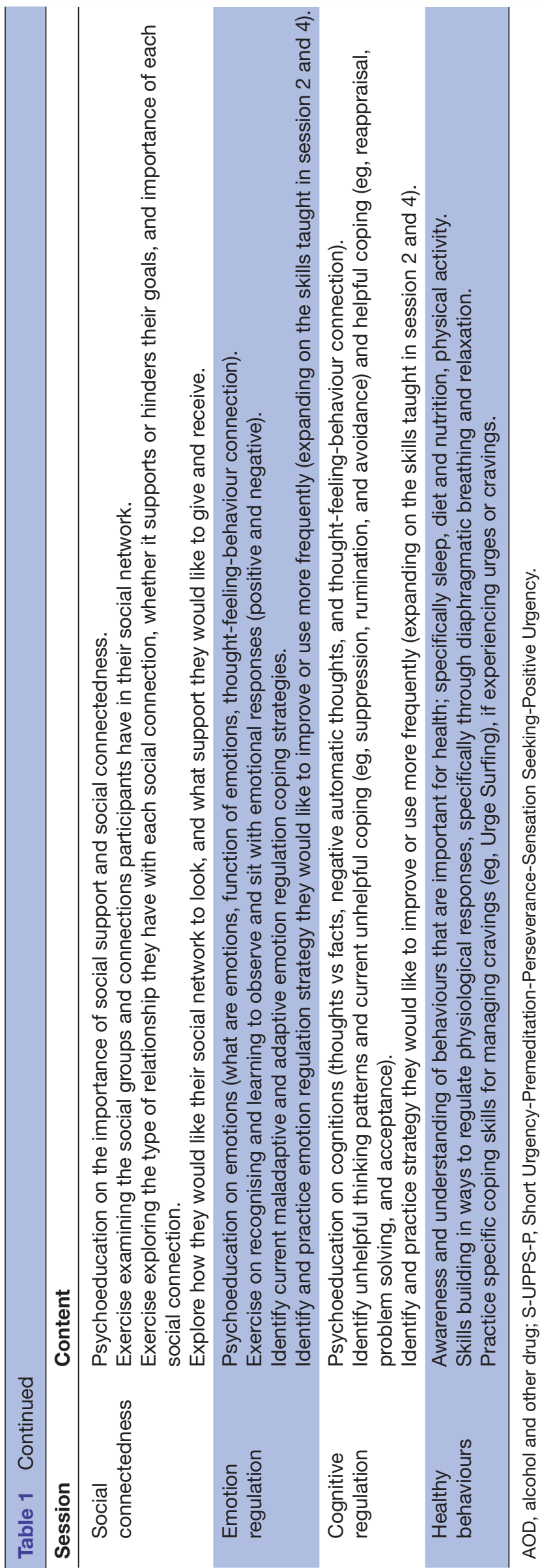

Primary outcomes

Alcohol and drug use

The WHO ASSIST ${ }^{55}$ screens for problem/risky substance use, covering tobacco, alcohol, cannabis, cocaine, amphetamine-type stimulants (including ecstasy) inhalants, sedatives, hallucinogens, opiates and 'other drugs'. A risk score is provided for each substance, and scores are grouped into low, moderate or high risk, which is then used to determine the level of intervention. The ASSIST has been found to be a reliable screening measure for psychoactive substance use and related problems. ${ }^{55}$ The Opiate Treatment Index (OTI) ${ }^{64}$ is also collected to measure of the frequency and quantity of alcohol, tobacco and illicit drug use in the past month, based on their last three occasions of use.

Mental health

Depressive symptoms are assessed using the PHQ-9. ${ }^{56}$ This valid nine-item self-report measure assesses depressive symptoms in the past 2 weeks, and is suitable for use in both primary care settings and over the phone. ${ }^{65}$ Anxiety is assessed using the GAD- $7,{ }^{57}$ which has been validated across a number of populations and will be suitable for assessing anxiety in young adults. ${ }^{66}$

\section{Secondary AOD outcomes}

Drinking self-efficacy

An adapted version of the 15-item Controlled Drinking Self-Efficacy Scale (CDSE) will be used to measure drinking and substance use self-efficacy. ${ }^{67}$ The CDSE provides a reliable and valid measure of self-efficacy to resist excessive drinking or substance use. Clients report how confident they are that they could resist the urge to drink heavily, or to engage in use of another drug, in each situation on a ranging from 0 (not at all confident) to 100 (very confident).

AOD-related problems

BriefYoung Adult Alcohol and other Drugs Consequences Questionnaire adapted from Kahler, Strong ${ }^{68}$ will be used to assess AOD-related problems in different domains (eg, social, medical, legal, family, vocational).

\section{Secondary well-being outcomes \\ Coping}

The Emotion-Oriented and Task-Oriented subscales from the Coping Inventory for Stressful Situations scale will be used to measure emotion and task orientated coping. ${ }^{69}$

\section{Well-being}

The brief 14-item version of the Mental Health ContinuumShort Form (MHC-SF) ${ }^{70}$ will ask participants about their emotional, psychological and social well-being in the past month. The MHC-SF has been found to be reliable and valid in young adults. ${ }^{31}$

Quality of life

The eight-item EUROHIS-QOL will collect information about the patient's quality of life (QOL) across a number 


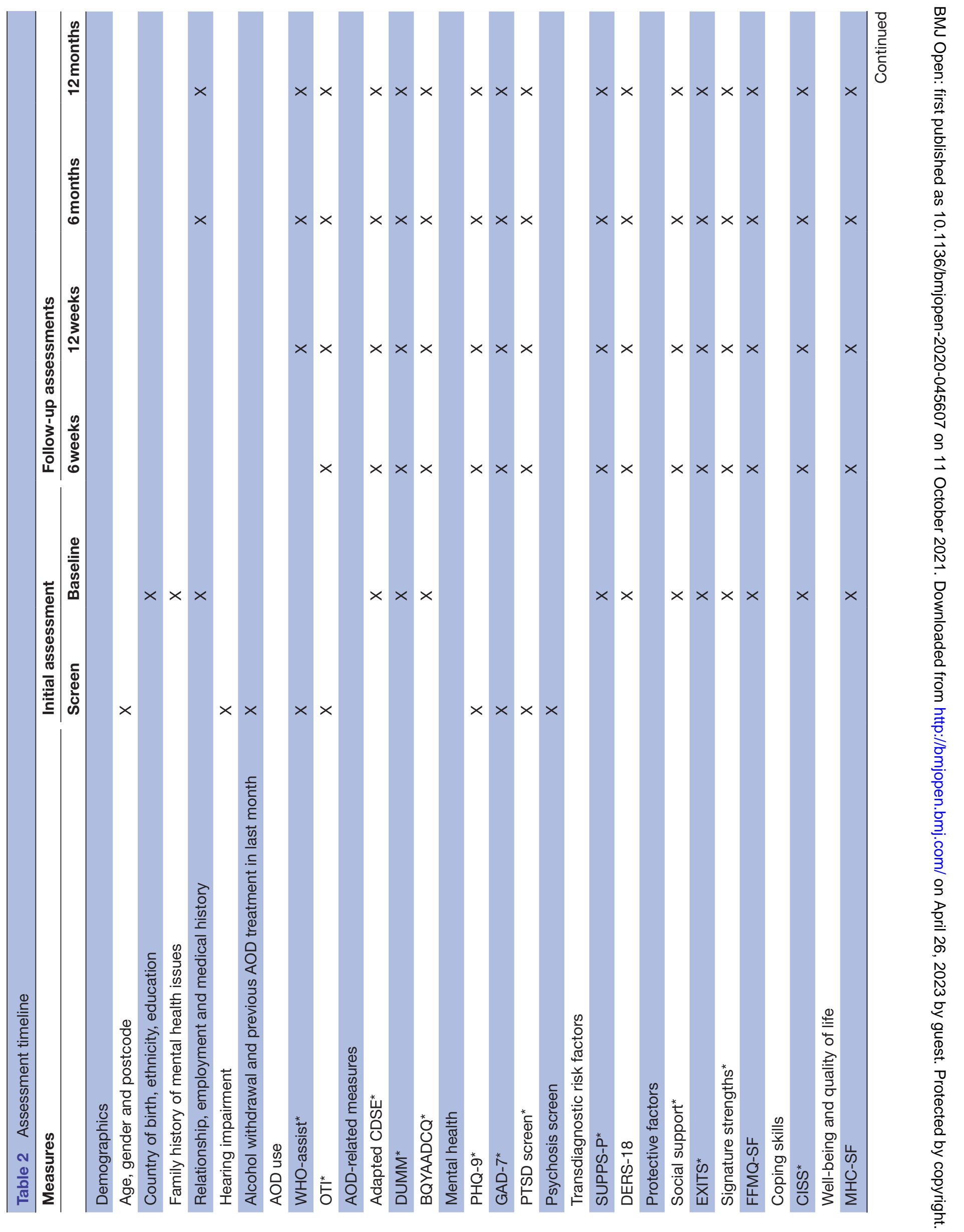




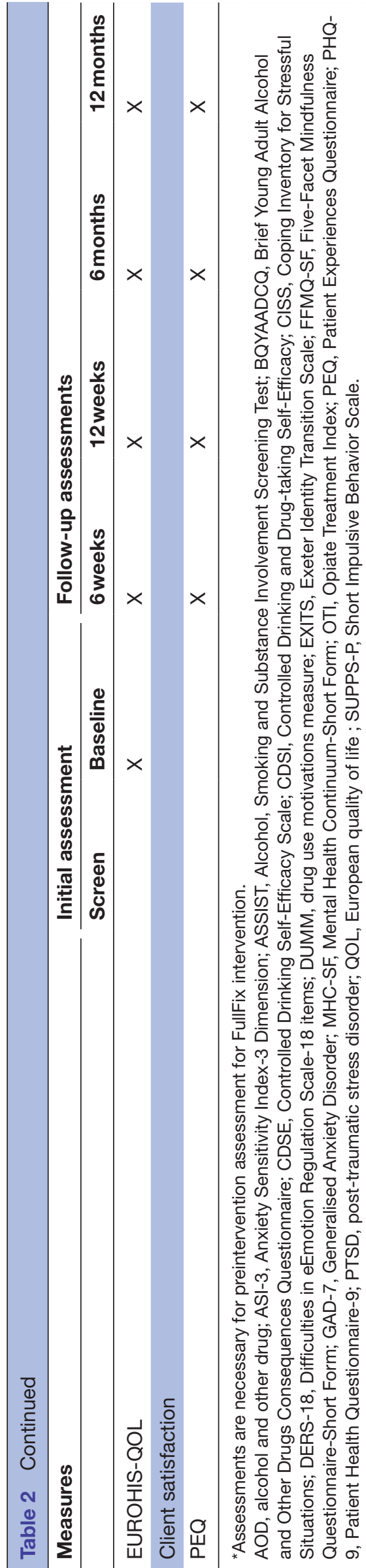

of domains. The EUROHIS-QOL has previously been tested with Australian populations and found to be a reliable and valid measure. ${ }^{71}$

\section{Transdiagnostic risk factors}

Impulsivity

Impulsivity will be measured using the Short UrgencyPremeditation-Perseverance-Sensation Seeking-Positive Urgency Scale. ${ }^{72}$ This 20-item measure provides subscores for the five subtraits of impulsivity-negative urgency, positive urgency, lack of perseverance, lack of premeditation and sensation seeking.

\section{Emotion dysregulation}

The 18-item Difficulties in Emotion Regulation Scale will be used to measure emotion regulation difficulties. The measure has good reliability and validity, and has been validated for use in young adults. ${ }^{73}$

\section{Drinking/drug use motives}

The 20-item Drinking Motives Questionnaire ${ }^{74}$ measures four different types of motives related to AOD use (social, coping, enhancement and social pressure and conformity). This study uses a modified version of this scale, the Drug Use Motive measure, to assess motives for AOD use.

\section{Protective factors}

\section{Social factors}

Social support will be measured using a four-item social support scale. ${ }^{75}$ To assess the group membership aspect of social belonging, an adapted version of the Exeter Identity Transition Scale will be used, ${ }^{76}$ which assesses number of important group memberships.

\section{Strengths}

To assess self-perceived strengths, an adaptation of the 24-item Signature Strengths Survey will be used. ${ }^{77}$ This measures captures the degree to which a strength is seen as defining to the self or not, and the self-rated top strengths (essential strengths) a person possesses.

\section{Self-regulation}

The Momentary Self-Regulation Questionnaire will be used to assess recent experience of self-regulation (Kim SJ, Scherer EA, Metcalf SA, et al. Can we measure momentary changes in self-regulation?: the development and validation of a novel momentary self-regulation scale. Manuscript in preparation 2019.). It is comprised of 12 items, rated on a 5-point Likert scale (1: not at all-5: extremely). The scale assess regulation across four domains: emotion dysregulation (eg, 'If I've felt negative emotions, I've made sure not to express them'), risk-taking behaviour ('I've quite enjoyed taking risks'), perserverance in goal-directed behaviour ('I've worked on what I planned until I succeeded'), and being mindful of the present moment ('I've found myself doing things without paying attention'-reversed). 


\section{Treatment satisfaction}

To measure participants' experiences with clinicians, the Patients Experience Questionnaire from the Improving Access to Psychological Therapies programme will be used at post-treatment assessment time-points. ${ }^{78}$ The scale is five items measuring client experiences on a 5-point Likert scale (1: strongly disagree to 5: strongly agree). Open ended responses will also be included to collect feedback from the participants about their level of satisfaction with their treatment and to better inform treatment procedures.

\section{Treatment fidelity}

Clinicians for the FullFix condition are registered psychologists on the research team, with a master's degree in clinical psychology. Training and supervision for FullFix will be conducted throughout the trial by registered clinical psychology supervisors. To assess intervention adherence and fidelity, telephone treatment sessions will be audiorecorded, with the participant's consent. Clinicians will also record a session component checklist for each session completed. Supervision meetings will be held fortnightly to monitor session delivery and treatment adherence. Additionally, the supervisor will review a randomly selected session audio segment, independently rating it and a Session Component Checklist, and discuss any departures from protocol. A random sample (20\%) of session recordings will be independently rated for treatment fidelity to ensure core features of the allocated treatment are delivered.

\section{Statistical analyses}

Feasibility and acceptability outcomes will be assessed using descriptive statistics of rates of eligibility, enrolment, attrition, reasons for refusal and follow-up rates. Independent means t-tests or non-parametric equivalents (for non-normal data) and $\chi^{2}$ tests will be used to compare treatment completers and non-completers. Responses to open ended questions will be evaluated using content and thematic analysis, as appropriate based on the nature of responses, to identify themes and patterns across responses.

The independent variable is treatment group, with two levels: (1) FullFix +Standard AOD care and (2) Standard AOD care alone. The primary outcome variables are AOD use and related harm (ASSIST, OTI) and depression/ anxiety symptoms (PHQ-9, GAD-7). Secondary outcomes include well-being (MHC-SF), quality of life (EUROHIS-QOL), drug taking confidence and coping selfefficacy to resist using alcohol heavily (adapted CDSE). A series of mixed effects, repeated measures analyses of variance (with time as the within subjects factor and group as the between subjects factor) will be employed to determine whether there are treatment group differences in improvements in primary and secondary outcomes of young people at 6weeks, 3-month, 6-month and 12-month follow-up compared with baseline. Analyses will be conducted on an intention-to-treat basis with all participants included in analyses, regardless of whether they withdrew from the treatment programme or did not complete all follow-up assessments. Analyses will be adjusted for covariates (eg, demographic characteristics) and factors associated with missing data. Bootstrapping will be used to account for any violations in the assumption of normality of residuals.

Moderators of treatment effects and process outcome variables include transdiagnostic risk and protective factors (emotion regulation, impulsivity, social support, coping skills and self-perceived strengths). To identify mechanisms and moderators of change and the strength of the factors involved, both multilevel models and structural equation models (using analyses in $R$ ) will be used for mediation and moderation analyses.

\section{Ethics and dissemination}

Ethical approval for this trial was provided by the University of Queensland (\#2018001185). Safety and risk management protocols attend to safety or urgent treatment issues. A clinical trials committee meets every 3 months to monitor the project's implementation, clinical and research integrity. Urgent issues are determined via email or telephone consultations and recorded to ensure consistency. Any young person who reports the presence of significant suicidal ideation/intent is provided with additional telephone support by a research psychologist and referral to additional treatment (through participating research sites or external local services), and an afterhours crisis team (if required). Any safety or urgent treatment issues will be managed as per usual safety and risk management procedures by the LLW clinicians. If a client screens positive for suicide risk during the screening procedure, their caseworker is made aware, and they are followed up by LLW treating staff.

\section{Dissemination}

The results of the trial will be disseminated through publication in peer-reviewed scientific journal, scientific presentations at conferences and distributed via a report and presentations to the partner organisation.

\section{CONCLUSION}

The current paper reports the study protocol of a pilot RCT determining the feasibility and efficacy of a new telehealth transdiagnostic CBT intervention for young people aged up to 35 years, presenting to AOD treatment services.

Identifying treatments that can address comorbidities within substance use settings that are accessible and feasible is of high importance considering the (1) low rates of treatment among young-people, (2) high rates of comorbidity, (3) poorer outcomes and (4) social and economic burden of comorbidity. Results of this study are expected to increase access to evidence-based and costeffective care for young people with comorbid AOD use and mental health problems. 
Author affiliations

${ }^{1}$ School of Psychology, The University of Queensland, Brisbane, Queensland, Australia

${ }^{2}$ National Centre for Youth Substance Use Research, The University of Queensland, Brisbane, Queensland, Australia

${ }^{3}$ School of Medicine and Public Health, The University of Newcastle, Callaghan, New South Wales, Australia

${ }^{4}$ Rural Clinical School, The University of Queensland, Toowoomba, Queensland, Australia

\section{Twitter Genevieve Dingle @Genevieve132}

Acknowledgements We acknowledge the partner organisation (Lives Lived Well) and the LLW trial sites and clinicians involved in the study. We would also like to acknowledge Mr James Curtain, who contributed to oversight of the trial, research design, recruitment strategy, and implementation of research findings into practice. We acknowledge all the young people who were involved in the trial.

Contributors LH conceived of the idea and had leadership and responsibility for the overall project. She contributed to research design, and protocol development. ZW contributed to research design, implementation of the project at service sites and project management, protocol development. NP, CAQ and DDA contributed to the execution of the program of research, coordination of activities and clinical supervision of staff and Research Assistants. GD, ALB, MT and AB were involved in the design and guiding the overall direction of the programme of research.All authors provided feedback on the manuscript.

Funding CAQ, DDA and ZW's research fellow positions are funded through an industry partnership between The University of Queensland and Lives Lived Well. LH is funded by a National Health and Medical Research Council Senior Research Fellowship (APP1119098). She also holds the industry-supported Lives Lived Well Chair in Alcohol, Drugs and Mental Health. Funding was provided for the trial by Australian Rotary Health Research Fund (2018001856). ALB is funded by a Nationa Health and Medical Research Council Senior Research Fellowship (APP1135901).

Disclaimer The funders had no role in the design of research, analyses, or decision to publish.

Competing interests None declared.

Patient consent for publication Not applicable.

Provenance and peer review Not commissioned; externally peer reviewed.

Supplemental material This content has been supplied by the author(s). It has not been vetted by BMJ Publishing Group Limited (BMJ) and may not have been peer-reviewed. Any opinions or recommendations discussed are solely those of the author(s) and are not endorsed by BMJ. BMJ disclaims all liability and responsibility arising from any reliance placed on the content. Where the content includes any translated material, BMJ does not warrant the accuracy and reliability of the translations (including but not limited to local regulations, clinical guidelines, terminology, drug names and drug dosages), and is not responsible for any error and/or omissions arising from translation and adaptation or otherwise.

Open access This is an open access article distributed in accordance with the Creative Commons Attribution Non Commercial (CC BY-NC 4.0) license, which permits others to distribute, remix, adapt, build upon this work non-commercially, and license their derivative works on different terms, provided the original work is properly cited, appropriate credit is given, any changes made indicated, and the use is non-commercial. See: http://creativecommons.org/licenses/by-nc/4.0/.

\section{ORCID iDs}

Zoe Walter http://orcid.org/0000-0001-8310-4021

Nina Pocuca http://orcid.org/0000-0003-0264-1680

\section{REFERENCES}

1 Kessler RC, Chiu WT, Demler O, et al. Prevalence, severity, and comorbidity of 12-month DSM-IV disorders in the National comorbidity survey replication. Arch Gen Psychiatry 2005;62:617-27.

2 Couwenbergh C, van den Brink W, Zwart K, et al. Comorbid psychopathology in adolescents and young adults treated for substance use disorders: a review. Eur Child Adolesc Psychiatry 2006;15:319-28.

3 Degenhardt L, Hall W, Lynskey M. Alcohol, cannabis and tobacco use among Australians: a comparison of their associations with other drug use and use disorders, affective and anxiety disorders, and psychosis. Addiction 2001;96:1603-14.

4 Grant BF, Stinson FS, Dawson DA, et al. Prevalence and cooccurrence of substance use disorders and independent mood and anxiety disorders: results from the National epidemiologic survey on alcohol and related conditions. Arch Gen Psychiatry 2004;61:807-16.

5 Prior K, Mills K, Ross J, et al. Substance use disorders comorbid with mood and anxiety disorders in the Australian general population. Drug Alcohol Rev 2017;36:317-24.

6 Kingston REF, Marel C, Mills KL. A systematic review of the prevalence of comorbid mental health disorders in people presenting for substance use treatment in Australia. Drug Alcohol Rev 2017;36:527-39.

7 Teesson M, Slade T, Mills K. Comorbidity in Australia: findings of the 2007 national survey of mental health and wellbeing. Aust N Z J Psychiatry 2009;43:606-14.

8 Lubman DI, Allen NB, Rogers N, et al. The impact of co-occurring mood and anxiety disorders among substance-abusing youth. J Affect Disord 2007;103:105-12.

9 Kessler RC, Nelson CB, McGonagle KA, et al. The epidemiology of co-occurring addictive and mental disorders: implications for prevention and service utilization. Am J Orthopsychiatry 1996;66:17-31.

10 Baker KD, Lubman DI, Cosgrave EM, et al. Impact of co-occurring substance use on 6 month outcomes for young people seeking mental health treatment. Aust N Z J Psychiatry 2007;41:896-902.

11 Salom CL, Betts KS, Williams GM, et al. Do young people with comorbid mental and alcohol disorders experience worse behavioural problems? Psychiatry Res 2014;219:372-9.

12 Lubman DI, Garfield JBB, Manning V, et al. Characteristics of individuals presenting to treatment for primary alcohol problems versus other drug problems in the Australian patient pathways study. BMC Psychiatry 2016;16:250.

13 Priester MA, Browne T, lachini A, et al. Treatment access barriers and disparities among individuals with co-occurring mental health and substance use disorders: an integrative literature review. J Subst Abuse Treat 2016;61:47-59.

14 Institute for Health Metrics and Evaluation. GBD compare data visualization, 2016. Available: http://vizhub.healthdata.org/gbdcompare

15 Rehm J, Shield KD. Global burden of disease and the impact of mental and addictive disorders. Curr Psychiatry Rep 2019;21:10.

16 Erskine HE, Moffitt TE, Copeland WE, et al. A heavy burden on young minds: the global burden of mental and substance use disorders in children and youth. Psychol Med 2015;45:1551-63.

17 Kessler RC, Amminger GP, Aguilar-Gaxiola S, et al. Age of onset of mental disorders: a review of recent literature. Curr Opin Psychiatry 2007;20:359-64.

18 Chapman C, Slade T, Hunt C, et al. Delay to first treatment contact for alcohol use disorder. Drug Alcohol Depend 2015;147:116-21.

19 Minkoff K. Developing standards of care for individuals with cooccurring psychiatric and substance use disorders. Psychiatr Serv 2001;52:597-9.

20 Kavanagh DJ, Mueser KT, Baker A. Management of comorbidity. In: Teesson M, Proudfoot H, eds. Comorbid mental disorders and substance use disorders: epidemiology prevention and treatment. Canberra, Australia: Commonwealth of Australia, 2003.

21 Hakobyan S, Vazirian S, Lee-Cheong S, et al. Concurrent disorder management guidelines. systematic review. Journal of Clinical Medicine 2020;9:2406.

22 Vujanovic AA, Meyer TD, Heads AM, et al. Cognitive-Behavioral therapies for depression and substance use disorders: an overview of traditional, third-wave, and transdiagnostic approaches. Am J Drug Alcohol Abuse 2017;43:402-15.

23 Reavley NJ, Cvetkovski S, Jorm AF, et al. Help-Seeking for substance use, anxiety and affective disorders among young people: results from the 2007 Australian national survey of mental health and wellbeing. Aust N Z J Psychiatry 2010;44:729-35.

24 Brown A, Rice SM, Rickwood DJ, et al. Systematic review of barriers and facilitators to accessing and engaging with mental health care among at-risk young people. Asia Pac Psychiatry 2016;8:3-22.

25 Deloitte. Mobile consumer survey 2018 the Australian cut, in mobile consumer survey. Australia: Deloitte, 2018.

26 Coughtrey AE, Pistrang N. The effectiveness of telephone-delivered psychological therapies for depression and anxiety: a systematic review. J Telemed Telecare 2018;24:65-74.

27 Turner C, Heyman I, Futh A, et al. A pilot study of telephone cognitive-behavioural therapy for obsessive-compulsive disorder in young people. Behav Cogn Psychother 2009;37:469-74. 
28 Muller I, Yardley L. Telephone-delivered cognitive behavioural therapy: a systematic review and meta-analysis. J Telemed Telecare 2011;17:177-84.

29 Sloan DM, Gallagher MW, Feinstein BA, et al. Efficacy of telehealth treatments for posttraumatic stress-related symptoms: a metaanalysis. Cogn Behav Ther 2011;40:111-25.

30 Hides L, Kavanagh DJ, Daglish M, et al. The Quik fix study: a randomised controlled trial of brief interventions for young people with alcohol-related injuries and illnesses accessing emergency department and crisis support care. BMC Emerg Med 2014;14:19.

31 Hides L, Wilson H, Quinn C, et al. QuikFix: enhanced motivational interviewing interventions for youth substance use. Adv Dual Diagn 2016;9:53-65.

32 Ehrenreich-May J, Chu BC. Transdiagnostic treatments for children and adolescents: principles and practice. New York: Guilford publications, 2013

33 Marchette LK, Weisz JR. Practitioner review: empirical evolution of youth psychotherapy toward transdiagnostic approaches. J Child Psychol Psychiatry 2017;58:970-84.

34 Aldao A, Gee DG, De Los Reyes A, et al. Emotion regulation as a transdiagnostic factor in the development of internalizing and externalizing psychopathology: current and future directions. Dev Psychopathol 2016;28:927-46.

35 Beauchaine TP. Future directions in emotion dysregulation and youth psychopathology. J Clin Child Adolesc Psychol 2015;44:875-96.

36 Eaton NR, Rodriguez-Seijas C, Carragher N, et al. Transdiagnostic factors of psychopathology and substance use disorders: a review. Soc Psychiatry Psychiatr Epidemiol 2015;50:171-82.

37 Shadur JM, Lejuez CW. Adolescent substance use and comorbid psychopathology: emotion regulation deficits as a Transdiagnostic risk factor. Curr Addict Rep 2015;2:354-63.

38 Hussong AM, Jones DJ, Stein GL, et al. An internalizing pathway to alcohol use and disorder. Psychol Addict Behav 2011;25:390-404.

39 Coyne JC, Downey G. Social factors and psychopathology: stress, social support, and coping processes. Annu Rev Psychol 1991;42:401-25.

40 Kaiser AJ, Milich R, Lynam DR, et al. Negative urgency, distress tolerance, and substance abuse among college students. Addict Behav 2012;37:1075-83.

41 Ruiz MA, Pincus AL, Schinka JA. Externalizing pathology and the five-factor model: a meta-analysis of personality traits associated with antisocial personality disorder, substance use disorder, and their co-occurrence. J Pers Disord 2008;22:365-88.

42 Smith GT, Cyders MA. Integrating affect and impulsivity: the role of positive and negative urgency in substance use risk. Drug Alcohol Depend 2016;163 Suppl 1: :S3-12.

43 Dingle GA, Neves DdaC, Alhadad SSJ, et al. Individual and interpersonal emotion regulation among adults with substance use disorders and matched controls. Br J Clin Psychol 2018;57:186-202.

44 Loxton NJ, Bunker RJ, Dingle GA, et al. Drinking not thinking: a prospective study of personality traits and drinking motives on alcohol consumption across the first year of university. Pers Individ Dif 2015:79:134-9.

45 Sloan E, Hall K, Moulding R, et al. Emotion regulation as a transdiagnostic treatment construct across anxiety, depression, substance, eating and borderline personality disorders: a systematic review. Clin Psychol Rev 2017;57:141-63.

46 Stanton K, Rozek DC, Stasik-O'Brien SM, et al. A transdiagnostic approach to examining the incremental predictive power of emotion regulation and basic personality dimensions. J Abnorm Psychol 2016;125:960-75.

47 Chan A-W, Tetzlaff JM, Altman DG, et al. Spirit 2013 statement: defining standard protocol items for clinical trials. Ann Intern Med 2013;158:200-7.

48 Eysenbach G, CONSORT-EHEALTH Group. CONSORT-EHEALTH: improving and standardizing evaluation reports of web-based and mobile health interventions. J Med Internet Res 2011;13:e126.

49 Patton KA, Gullo MJ, Connor JP, et al. Social cognitive mediators of the relationship between impulsivity traits and adolescent alcohol use: identifying unique targets for prevention. Addict Behav 2018;84:79-85.

50 Mawson E, Best D, Beckwith M, et al. Social identity, social networks and recovery capital in emerging adulthood: a pilot study. Subst Abuse Treat Prev Policy 2015;10:45.

51 Newby JM, Twomey C, Yuan Li SS, et al. Transdiagnostic computerised cognitive behavioural therapy for depression and anxiety: a systematic review and meta-analysis. J Affect Disoro 2016;199:30-41.

52 Reinholt N, Krogh J. Efficacy of transdiagnostic cognitive behaviour therapy for anxiety disorders: a systematic review and meta-analysis of published outcome studies. Cogn Behav Ther 2014;43:171-84.
53 Norton PJ, Paulus DJ. Toward a unified treatment for emotional disorders: update on the science and practice. Behav Ther 2016;47:854-68.

54 Chiesa A, Serretti A. Are mindfulness-based interventions effective for substance use disorders? A systematic review of the evidence. Subst Use Misuse 2014;49:492-512.

55 WHO ASSIST Working Group. The alcohol, smoking and substance involvement screening test (assist): development, reliability and feasibility. Addiction 2002;97:1183-94.

56 Kroenke K, Spitzer RL, Williams JB. The PHQ-9: validity of a brief depression severity measure. J Gen Intern Med 2001;16:606-13.

57 Spitzer RL, Kroenke K, Williams JBW, et al. A brief measure for assessing generalized anxiety disorder: the GAD-7. Arch Intern Med 2006;166:1092-7.

58 Prins A, Ouimette P, Kimerling R, et al. The primary care PTSD screen (PC-PTSD): development and operating characteristics. Primary Care Psychiatry 2004;9:9-14.

59 Hides L, Carroll S, Catania L, et al. Outcomes of an integrated cognitive behaviour therapy (CBT) treatment program for cooccurring depression and substance misuse in young people. $J$ Affect Disord 2010;121:169-74.

60 Snow G. Blockrand, 2015. Available: https://cran.r-project.org/web/ packages/blockrand/blockrand.pdf

61 Hides L, Carroll S, Scott R, et al. Quik fix: a randomized controlled trial of an enhanced brief motivational interviewing intervention for alcohol/cannabis and psychological distress in young people. Psychother Psychosom 2013;82:122-4.

62 Hides L, Quinn C, Chan G, et al. Telephone-based motivational interviewing enhanced with individualised personality-specific coping skills training for young people with alcohol-related injuries and illnesses accessing emergency or rest/recovery services: a randomized controlled trial (QuikFix). Addiction 2021;116:474-84

63 Miller WR, Rollnick S. Motivational interviewing: helping people change. 3rd ed. New York, NY: Guilford Press, 2013.

64 Darke S, Hall W, Wodak A, et al. Development and validation of a multi-dimensional instrument for assessing outcome of treatment among opiate users: the opiate treatment index. Br J Addict 1992;87:733-42

65 Cameron IM, Crawford JR, Lawton K, et al. Psychometric comparison of $\mathrm{PHQ}-9$ and HADS for measuring depression severity in primary care. Br J Gen Pract 2008;58:32-6.

66 Kroenke K, Spitzer RL, Williams JBW, et al. The patient health questionnaire somatic, anxiety, and depressive symptom scales: a systematic review. Gen Hosp Psychiatry 2010;32:345-59.

67 Sitharthan T, Job RFS, Kavanagh DJ, et al. Development of a controlled drinking self-efficacy scale and appraising its relation to alcohol dependence. J Clin Psychol 2003;59:351-62.

68 Kahler CW, Strong DR, Read JP. Toward efficient and comprehensive measurement of the alcohol problems continuum in college students: the brief young adult alcohol consequences questionnaire. Alcohol Clin Exp Res 2005;29:1180-9.

69 Cohan SL, Jang KL, Stein MB. Confirmatory factor analysis of a short form of the coping inventory for stressful situations. J Clin Psychol 2006;62:273-83.

70 Keyes CL. The subjective well-being of America's youth: toward a comprehensive assessment. Adolescent \& Family Health 2006;4:3-11.

71 Schmidt S, Mühlan H, Power M. The EUROHIS-QOL 8-item index: psychometric results of a cross-cultural field study. Eur $J$ Public Health 2006;16:420-8.

72 Cyders MA, Littlefield AK, Coffey S, et al. Examination of a short English version of the UPPS-P impulsive behavior scale. Addict Behav 2014;39:1372-6.

73 Victor SE, Klonsky ED. Validation of a brief version of the difficulties in emotion regulation scale (DERS-18) in five samples. $J$ Psychopathol Behav Assess 2016;38:582-9.

74 Cooper ML. Motivations for alcohol use among adolescents: development and validation of a four-factor model. Psychol Assess 1994:6:117-28.

75 Gottlieb BH, Bergen AE. Social support concepts and measures. J Psychosom Res 2010;69): :511-20.

76 Haslam C, Holme A, Haslam SA, et al. Maintaining group memberships: social identity continuity predicts well-being after stroke. Neuropsychol Rehabil 2008;18:671-91.

77 McGrath RE. Technical report: the VIA assessment suite for adults: development and evaluation. Cincinnati, OH: VIA Institute on Character, 2017

78 National health service. Improving access to psychological therapies (IAPT), the IAPT data Handook, National health service, editor. London, UK: National health service, 2011. https://webarchive. nationalarchives.gov.uk/ 